\title{
A facile method for isolation of recombinant human apolipoprotein A-I from E. coli
}

Nikita Ikon ${ }^{a}$, Jennifer Shearer ${ }^{a}$, Jianfang Liu ${ }^{b}$, Jesse J. Tran ${ }^{c}$, ShiBo Feng ${ }^{d}$, Ayako Kamei $^{\mathrm{a}}$, Jennifer A. Beckstead ${ }^{\mathrm{a}}$, Robert S. Kiss ${ }^{\mathrm{d}}$, Paul M. Weers ${ }^{\mathrm{c}}$, Gang Ren ${ }^{\mathrm{b}}$, and Robert O. Ryan ${ }^{\mathrm{a} *}$

${ }^{a}$ Children's Hospital Oakland Research Institute 5700 Martin Luther King Jr. Way, Oakland CA94609 USA

${ }^{\mathrm{b}}$ The Molecular Foundry, Lawrence Berkeley National Laboratory, Berkeley CA94720 USA

${ }^{\mathrm{C}}$ Department of Chemistry and Biochemistry, California State University Long Beach 1250 Bellflower Boulevard, Long Beach, CA 90840USA

${ }^{\mathrm{d}}$ Research Institute of the McGill University Health Centre Glen site, EM1.2220 1001 boul Decarie, Montreal, QCH4A 3J1 Canada

*To whom correspondence should be addressed, rryan@chori.org 


\begin{abstract}
Apolipoprotein (apo) A-I is the major protein component of high-density lipoprotein (HDL) and plays key roles in the Reverse Cholesterol Transport pathway. In the past decade, reconstituted HDL ( $\mathrm{rHDL}$ ) has been employed as a therapeutic agent for treatment of atherosclerosis. The ability of rHDL to promote cholesterol efflux from peripheral cells has been documented to reduce the size of atherosclerotic plaque lesions. However, development of apoA-I rHDL-based therapeutics for human use requires a cost effective process to generate an apoA-I product that meets "Good Manufacturing Practice" standards. Methods available for production and isolation of unmodified recombinant human apoA-I at scale are cumbersome, laborious and complex. To overcome this obstacle, a streamlined two-step procedure has been devised for isolation of recombinant untagged human apoA-I from E. coli that takes advantage of its ability to re-fold to a native conformation following denaturation. Heat treatment of a sonicated $E$. coli supernatant fraction induced precipitation of a large proportion of host cell proteins (HCP), yielding apoA-I as the major soluble protein. Reversed-phase HPLC of this material permitted recovery of apoA-I largely free of HCP and endotoxin. Purified apoA-I possessed $\alpha$-helix secondary structure, formed $\mathrm{rHDL}$ upon incubation with phospholipid and efficiently promoted cholesterol efflux from cholesterol loaded J774 macrophages.
\end{abstract}

Key words: Apolipoprotein A-I; high density lipoprotein; thermal denaturation; cholesterol efflux; circular dichroism; E. coli; reversed phase HPLC 


\section{Introduction}

Apolipoprotein (apo) A-I is the major protein component of plasma high-density lipoprotein (HDL). The ability of isolated apoA-I to induce self-assembly of discoidal reconstituted $\mathrm{HDL}$ ( $\mathrm{rHDL}$ ) in vitro has led to numerous applications. For example, apoAI rHDL have been administered to patients as therapy for cardiovascular disease [1,2]. Others have employed apoA-I mimetics as a stabilizing scaffold for miniature membranes that allow assimilation of transmembrane proteins in a native-like conformation [3]. In yet other applications, apoA-I serves as an integral component of "nanodisk" delivery particles $[4,5]$ or as a structural component of rHDL harboring contrast agents for magnetic resonance imaging [6].

Further development and broader use of this rapidly advancing technology, as well as potential commercialization of $\mathrm{rHDL}$ based therapeutics, requires a robust supply of high purity apoA-I. Whereas research scale production and downstream processing of recombinant apoA-I is simplified by appending cleavable tags onto the protein [7 - 9], these extraneous sequences must be removed prior to use in therapeutic agents intended for humans. At the same time, production and isolation of untagged recombinant proteins in Escherichia coli is oftentimes plagued by contamination from host cell protein (HCP) and endotoxin [10]. Contaminating HCP in a biopharmaceutical drug can act as adjuvants or be immunogenic [11]. Thus, E. coli HCP present a safety concern such that, typically, their levels are reduced to the $\mathrm{ng} / \mathrm{mg}$ range in recombinant therapeutic proteins. Current methods to achieve this require multiple chromatography steps, often conducted in the presence of chaotropic salts. A complementary, but painstaking, strategy involves the use of genetic engineering to delete genes encoding HCP contaminants [10].

One of the key intrinsic properties of apoA-I is structural resilience. Indeed, it is well known that apoA-I refolds to a native state following thermal denaturation [12, 13]. In the present study, this property is exploited to isolate mature recombinant WT human apoA-I from $E$. coli without the use of affinity tags or cumbersome and laborious chromatographic steps. The method described is a facile two-step purification scheme 
that yields highly purified apoA-I that retains native structure-function properties with minimal contamination by HCP or endotoxin.

\section{Material and Methods}

ApoA-I cDNA cloning and bacterial transformation. A cDNA encoding mature human apoA-I (243 amino acids) was cloned into a pET-22b(+) vector at Ndel and Notl restriction sites. The insert contained a stop codon at the final position to prevent expression of a vector encoded downstream His-tag. The assembled plasmid was transformed into $E$. coli $\mathrm{DH} 5 \alpha$ cells and cultured on ampicillin-infused agar plates to select transformed clones. Colonies were picked and cultured for plasmid preparation. DNA sequencing was performed on the plasmid vector to confirm the desired insertion was achieved.

Expression of recombinant human apoA-I. The apoA-I pET-22b plasmid vector was used to transform BLR(DE3) competent E. coli cells (Novagen Inc) by heat shock (BL21 and BLR cells were also tested but resulted in lower yield). Transformed cells were grown overnight in NZCYM media (Sigma-Aldrich) supplemented with $50 \mu \mathrm{g} / \mathrm{mL}$ ampicillin (Sigma-Aldrich) at $37^{\circ} \mathrm{C}$ with shaking (200 rpm). Overnight cultures were used to inoculate $1 \mathrm{~L}$ of NZCYM /ampicillin media followed by culturing at $37^{\circ} \mathrm{C}$ and $200 \mathrm{rpm}$ until the $\mathrm{OD}_{600}=0.7$. Isopropyl $\beta-D-1$-thiogalactopyranoside (Sigma-Aldrich) was added ( $0.5 \mathrm{mM}$ final concentration) to induce apoA-I expression and bacteria were cultured for a further $5 \mathrm{~h}$ at $25^{\circ} \mathrm{C}$ with shaking.

Purification procedure. Bacteria were pelleted by centrifugation, re-suspended in $10 \mathrm{~mL}$ phosphate buffered saline (PBS; $20 \mathrm{mM}$ sodium phosphate, $150 \mathrm{mM} \mathrm{NaCl}, \mathrm{pH}$ 7.2) per liter bacterial culture and disrupted by sonication on a Branson 450 sonicator fitted with a $1 \mathrm{~cm}$ tip probe. Ten $\mathrm{ml}$ suspensions were subjected to three rounds of sonication for $30 \mathrm{sec}$ each at $50 \%$ duty cycle (output level 5 ). The resulting cell lysate was centrifuged at $20,000 \mathrm{xg}$ for $30 \mathrm{~min}$ at $4^{\circ} \mathrm{C}$ to separate insoluble material. The supernatant fraction 
was collected and heated for $10 \mathrm{~min}$ at specified temperatures (range: $65^{\circ} \mathrm{C}-95^{\circ} \mathrm{C}$ ). Subsequently, the sample was cooled and centrifuged at 20,000xg for 30 min at $4^{\circ} \mathrm{C}$. The recovered supernatant was subjected to reversed-phase high performance liquid chromatography (HPLC) on a Perkin-Elmer Series 200 instrument. The sample was applied to a semi-preparative RXC-8 Zorbax 300SB column and eluted with a linear A/B gradient of $2 \%$ solvent $B$ per min, where solvent $A$ was $0.05 \%$ trifluoroacetic acid in water and solvent $B$ was $0.05 \%$ trifluoroacetic acid in acetonitrile. Absorbance was monitored at $230 \mathrm{~nm}$ and the peak corresponding to apoA-I collected, lyophilized and stored at $-20^{\circ} \mathrm{C}$ until use. As a control for comparison, His-tag apoA-I was expressed in $E$. coli and isolated by nickel chelation affinity chromatography according to Ryan et al [8].

Analytical procedures. Sodium dodecyl sulfate-polyacrylamide gel electrophoresis (SDS-PAGE) was performed using either a $10-20 \%$ acrylamide gradient tricine gel or a fixed $18 \%$ acrylamide slab gel and stained with GelCode Blue. For Western blotting, proteins were electrophoretically transferred to a PVDF membrane, probed with antiapoA-I (Meridian Life Science Inc., K45252G, 1:10,000 dilution) and visualized via enhanced chemiluminescence $(E C L)$ reagent. Protein concentration was determined by the bicinchoninic acid assay (Pierce Chemical Co.) with bovine serum albumin (BSA) as standard. Endotoxin (i.e. lipopolysaccharide; LPS) was quantified using the Limulus Amebocyte Lysate Kinetic-QCL assay (Lonza).

Far UV circular dichroism spectroscopy. Far UV circular dichroism (CD) spectroscopy was performed on a Jasco 810 spectropolarimeter. Scans were recorded between 185

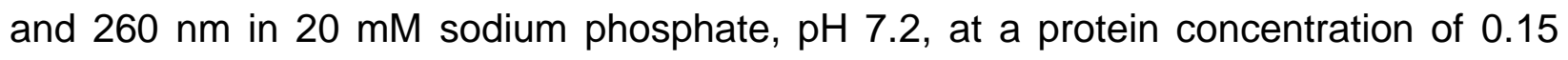
$\mathrm{mg} / \mathrm{mL}$ in a $1 \mathrm{~mm}$ path length circular cuvette. Secondary structure content was calculated as previously described [14]. For guanidine $\mathrm{HCl}$ denaturation experiments, protein samples $(0.2 \mathrm{mg} / \mathrm{mL})$ were incubated overnight at a given denaturant concentration to attain equilibrium, and ellipticity measured at $222 \mathrm{~nm}$ in a $1 \mathrm{~mm}$ path length cuvette. 
Cholesterol efflux assays. J774 macrophages were simultaneously cholesterol-loaded and radiolabeled by incubation with $25 \mu \mathrm{g} / \mathrm{mL}$ acetylated LDL containing $3 \mu \mathrm{Ci} / \mathrm{mL}$ $\left[{ }^{3} \mathrm{H}\right]$ cholesterol. Cells were washed with media containing $2 \mathrm{mg} / \mathrm{mL}$ BSA to remove unincorporated radioactivity and equilibrated overnight in media supplemented with BSA. After equilibration, cholesterol efflux assays were conducted by introducing $10 \mu \mathrm{g}$ of a specified apoA-I preparation to the cells and incubation for 1-24 h. At indicated times, the media was collected and radioactivity measured by liquid scintillation spectrometry (PerkinElmer). Subsequently, cells were treated with $0.5 \mathrm{~mol} / \mathrm{L} \mathrm{NaOH}$ for $12 \mathrm{~h}$ and residual cell-associated radioactivity determined. Percent efflux was computed from the $\left[{ }^{3} \mathrm{H}\right]$ cholesterol in media over total $\left[{ }^{3} \mathrm{H}\right]$ cholesterol (media + cell).

Statistical analysis. Statistical significance between groups was calculated using the two-tailed Student's t-test. Data are presented as mean \pm standard deviation $(n=3)$. Pvalues $<0.05$ were considered significant.

$r H D L$ formation. Five $\mathrm{mg}$ dimyristoylphosphatidylcholine (DMPC) was dissolved in chloroform / methanol (3:1 v/v) and dried under a stream of $\mathrm{N}_{2}$ gas, forming a thin film on the vessel wall. Residual organic solvent was removed under vacuum. The prepared lipid was then dispersed in $1 \mathrm{ml}$ PBS followed by the addition of $2 \mathrm{mg}$ apoA-I $(4 \mathrm{mg} / \mathrm{ml}$ stock solution in PBS). The sample was bath sonicated at $22^{\circ} \mathrm{C}$ for approximately 5 min until the turbid sample clarified.

Electron microscopy (EM). A freshly prepared untagged apoA-I rHDL sample was processed for negative staining as previously described [15 - 17]. In brief, the sample was diluted to $5 \mu \mathrm{g} / \mathrm{mL}$ protein with Dulbecco's PBS. An aliquot $(\sim 4 \mu \mathrm{L})$ of the sample was placed on a glow-discharged thin-carbon-coated 200 mesh copper grid (CF200-Cu, Electron Microscopy Sciences, Hatfield, PA and CU-200CN, Pacific grid-tech, San Francisco, CA). After $\sim 1$ min, excess solution was blotted with filter paper, the grid was washed with water, stained with $1 \%(\mathrm{w} / \mathrm{v})$ uranyl formate and dried under a stream of $\mathrm{N}_{2}$ gas. 
EM data acquisition and image processing. The negatively stained specimen was examined on a Zeiss Libra 120 Plus TEM (Carl Zeiss NTS, Oberkochen, Germany) operating at $120 \mathrm{kV}$ with $20 \mathrm{eV}$ in-column energy filtering at room temperature. Micrographs were acquired by a Gatan UltraScan 4Kx4K CCD at 80,000x magnification (each pixel corresponding to $1.48 \AA$ ) under near Scherzer focus $(0.1 \mu \mathrm{m})$ and defocus of $0.4 \mu \mathrm{m}$. Micrographs were processed with EMAN, SPIDER, and FREALIGN software packages as described elsewhere [17 - 20]. The contrast transfer function parameters of each micrograph were determined and corrected. A total of 2,986 rHDL were used for reference-free class averaging with the EMAN software package.

\section{Results}

Overview of strategy: Seeking an improved method for isolation of recombinant untagged human apoA-I from E. coli, we took advantage of the unique structural resilience of this protein. Naturally devoid of cysteine, biophysical studies $[12,13]$ have documented that apoA-I re-folds to its native conformation following denaturation (i.e. exposure to chaotropic salts or heat treatment). To incorporate this intrinsic property into an isolation scheme for recombinant apoA-I, the process described below was devised.

The effect of culture temperature on apoA-I expression. When recombinant WT human apoA-I was expressed in E. coli under standard conditions the bulk of apoA-I produced remained in the bacteria. Therefore, following expression, E. coli were pelleted by centrifugation, re-suspended in PBS, lysed by probe sonication and centrifuged. The corresponding supernatant and pellet fractions were collected and analyzed by SDS PAGE (Figure 1). When E. coli was transformed with empty vector no apoA-I was detected. When apoA-I expression was examined as a function of culture temperature, increased apoA-I was detected as the culture temperature was lowered from $37^{\circ} \mathrm{C}$ to $30^{\circ} \mathrm{C}$ to $25^{\circ} \mathrm{C}$. However, at culture temperatures of $20^{\circ} \mathrm{C}$ or $15^{\circ} \mathrm{C}$, apoA-I protein production was lower than that observed at $25^{\circ} \mathrm{C}$ (see Supplementary Figure S1). Figure 1 also revealed that the bulk of apoA-I is recovered in the supernatant fraction 
obtained after probe sonication of the bacterial cell lysate. Based on this data, all subsequent apoA-I expression studies were performed at $25^{\circ} \mathrm{C}$.

Step 1: Heat treatment. Despite the large amount of apoA-I present in the supernatant fraction, numerous HCP were also present. In an effort to selectively remove HCP, the supernatant was heated to various temperatures ranging from $65^{\circ} \mathrm{C}-95^{\circ} \mathrm{C}$ for $10 \mathrm{~min}$. After cooling and centrifugation to pellet insoluble material, SDS-PAGE was performed to assess the relative distribution of apoA-I between the supernatant and pellet fractions (Figure 2). At every temperature tested, large amounts of HCP, but little apoA-I, were recovered in the pellet fraction. Conversely, the supernatant fractions were greatly enriched in apoA-I. Heat exposure for longer time periods (30 min to $1 \mathrm{~h}$ ) resulted in significantly less apoA-I recovery in the supernatant fraction with only modest further reductions in HCP (Supplementary Figure S2). On the other hand, when the time of heat exposure was limited to $10 \mathrm{~min}$, increased temperature resulted in less HCP contamination in the supernatant with little change in apoA-I distribution. Thus, 10 min heat exposure at $95^{\circ} \mathrm{C}$ was adopted as the optimal procedure. Based on the relative enrichment of apoA-I following heat treatment, it was hypothesized that reversed-phase HPLC could achieve two goals: a) isolation of apoA-I and b) removal of bacteria-derived endotoxin from the preparation.

Step 2: Reversed-phase HPLC. The soluble fraction obtained following heat treatment of an E. coli cell lysate (Step 1) was subjected to reversed-phase HPLC. ApoA-I eluted as a single major peak at approximately $50 \%$ acetonitrile (solvent $\mathrm{B}$ ). The peak corresponding to apoA-I was collected, dialyzed into PBS and analyzed by SDS-PAGE (Figure 3, panel A). The stained gel indicates this chromatographic step removes the majority of HCP, resulting in a sample with higher apparent purity than a control His-tag apoA-I purified by nickel chelation chromatography. The yield of untagged apoA-I was $\sim 6 \mathrm{mg} /$ liter bacterial culture. A corresponding anti-apoA-I Western blot (panel B) revealed a single immune-reactive band at $28 \mathrm{kDa}$.

Endotoxin assay. Given the importance of minimizing endotoxin contamination for therapeutic and biotechnology related applications, bacteria-derived endotoxin levels 
were determined at various stages of the purification process (Figure 4). The initial heat treatment step led to a $\sim 10$-fold reduction in endotoxin levels relative to the starting material. This was similar to the degree of reduction observed following nickel chelation chromatography of control His-tag apoA-I. Following the reversed phase HPLC step, however, a further 100,000-fold decrease in endotoxin was achieved for an overall six orders of magnitude reduction, relative to starting material $(p<0.05)$. Endotoxin levels in the final preparation were $60.8 \pm 36.3 \mathrm{EU} / \mathrm{mL}(\mathrm{n}=3)$. Based on an average protein concentration of $5 \mathrm{mg} / \mathrm{ml}$, this corresponds to $\sim 12 \mathrm{EU} / \mathrm{mg}$ apoA-I.

Secondary structure content and stability of apoA-I. To determine if heat exposure results in a structurally damaged apoA-I product, far UV CD spectroscopy was performed (Figure 5). An $\alpha$-helix content of $48.0 \pm 1.7 \%$ was calculated for control Histag apoA-I and $53.3 \pm 1.7 \%$ for untagged apoA-I. The $\alpha$-helix contents observed for untagged apoA-I (heat exposed) and control His-tag apoA-I are in good agreement with values $(50 \% \alpha$-helix) reported by others [12]. Similarly, untagged apoA-I and His-tag apoA-I displayed a similar ability to resist guanidine $\mathrm{HCl}$-induced unfolding (midpoint of guanidine $\mathrm{HCl}$ induced denaturation $=0.97 \pm 0.03 \mathrm{M}$ for His-tag apoA-I and $1.03 \pm 0.03$ $M$ for untagged apoA-I). These data indicate the purification method involving heat exposure yields a protein that has not suffered irreparable loss of secondary structure content or intrinsic stability.

Cholesterol efflux capacity of isolated recombinant apoA-I. A major function of apoA-I is promotion of cholesterol efflux from cells via interaction with members of the ATP binding cassette transporter protein family. To determine if the ability of apoA-I to function as a cholesterol acceptor was compromised as a result of the purification scheme employed, cholesterol efflux activity was measured in $\mathrm{J} 774$ macrophages (Figure 6). Over a period of $24 \mathrm{~h}$ no significant differences in cholesterol efflux were observed between untagged apoA-I (heat exposed) and control His-tag apoA-I.

ApoA-I dependent formation of $r H D L$. A major therapeutic application for recombinant apoA-I involves formation of $r H D L$. To investigate the ability of apoA-I to form these 
complexes, untagged apoA-I was incubated with an aqueous dispersion of DMPC. Bath sonication of the apolipoprotein / phospholipid mixture at $22{ }^{\circ} \mathrm{C}$ induced sample clarification, indicating rHDL formation. Analysis by negative stain electron microscopy revealed a population of discoidal particles with diameters in the range of 12 to $20 \mathrm{~nm}$ (Figure 7). The particle diameter distribution and discoidal shape of the particles is similar to previously described apoA-I rHDL [4].

\section{Discussion}

There is a growing need for apoA-I for commercial applications in nanotechnology [21] and as a therapeutic [22]. While it is possible to isolate apoA-I from human blood products, this approach is costly and vulnerable to disease transmission [23 - 25]. Approaches to generate recombinant apoA-I using yeast, baculovirus or bacteria present their own challenges. Each of these systems has been utilized for apoA-I production. Feng et al [26] reported a bio-reactor scale Pichia pastoris-based expression system that utilized a purification scheme involving acetone precipitation and ion exchange chromatography. Sorci-Thomas et al. [27] and Pyle et al [28] developed baculovirus systems capable of expressing apoA-I. Drawbacks include the length of time to express the protein and downstream processing complexity. Methods employing bacteria generally append tags or extensions onto the target protein. While this approach streamlines processing and purification, it requires additional steps, and increased cost, to remove the tag. In addition, leftover amino acids and/or non-specific cleavage can present problems $[7-9,29]$. In the present approach we elected to express mature untagged human apoA-I. Although apoA-I produced in this manner is contaminated with HCP, by taking advantage of the unique ability of the class of exchangeable apolipoproteins to refold following thermal denaturation $[12,13,30]$ it was possible to eliminate the majority of HCP by heat treatment, while maintaining apoA-I in solution. The folding $\rightleftarrows$ unfolding transition of apoA-I has its midpoint between 50 and $60{ }^{\circ} \mathrm{C}$ and is readily measured by CD spectroscopy. Far UV CD analysis revealed the $\alpha-$ helix content of untagged apoA-I recovers fully following exposure to temperatures as 
high as $95^{\circ} \mathrm{C}$. At the same time, after a single $10 \mathrm{~min}$ heat exposure step, a large proportion of contaminating HCP precipitate. A subsequent semi-preparative reversedphase HPLC step yielded highly purified WT apoA-I. When the effect of bacterial culture temperature on apoA-I production was examined, enhanced apoA-I expression was observed upon lowering the culture temperature from $37^{\circ} \mathrm{C}$ to $25^{\circ} \mathrm{C}$. The reason for this is unknown but may be related to a temperature-dependent membrane damaging, detergent-like effect of apoA-I.

Although significant quantities of untagged apoA-I can be prepared using this two-step isolation protocol, we sought to assess functionality of the final protein product. The untagged apoA-I product resisted guanidine $\mathrm{HCl}$-induced denaturation to an extent similar to other apoA-I preparations [31], and cholesterol efflux assays revealed this apoA-I preparation is functional. Likewise, negative stain electron microscopy confirmed that apoA-I forms rHDL upon incubation with phospholipid.

A critical aspect related to use of recombinant apoA-I produced in $E$. coli is the extent to which it is contaminated by bacterial endotoxin. Indeed, LPS contamination is a major problem encountered in the use of bacteria in the manufacture of proteins. While affinity purification of His-tag apoA-I yielded a modest reduction in endotoxin contamination, the protocol outlined herein reduced endotoxin levels by one million-fold. Although the final preparation still contained detectable endotoxin levels, the amounts were close to Good Manufacturing Practice standards. Given that endotoxin limits for protein-based injectables are typically $0.5 \mathrm{EU} / \mathrm{mg}$, an additional endotoxin removal step(s) [32] may be required for use of recombinant apoA-I isolated by the present protocol in humans. Regardless, overall low endotoxin content together with scalability of the two-step process combines to makes this purification scheme an attractive option to produce WT apoA-I for commercial and pharmaceutical applications. Whereas scale up and yield optimization studies have not been pursued, the methods employed are readily amenable to bioreactor fermentation methods without compromising product purity. 


\section{Conclusions}

ApoA-I is an integral component of $\mathrm{rHDL}$ employed in a growing number of therapeutic and biotechnology applications. Herein, we report a two-step procedure for isolation of recombinant human apoA-I from $E$. coli without extraneous sequence tags. The method employed takes advantage of reversible folding $\rightleftarrows$ unfolding by apoA-I in response to thermal denaturation. The product apoA-I possesses $\alpha$-helix secondary structure, forms rHDL upon incubation with phospholipid, promotes cholesterol efflux and has low LPS contamination. Thus, the method described is amenable to production scale up and formulation into therapeutics for human use.

\section{Acknowledgements}

The authors thank Zainab Abusbeaa for technical assistance. Supported by a grant from NIH (R37 HL-64159). Work at the Molecular Foundry was supported by the US Department of Energy, Office of Basic Energy Sciences under Contract No. DE-AC0205CH11231. JL and GR acknowledge support from the NHLBI (HL115153) and the NIGMS (GM104427). NI acknowledges support from NIH Training Grant (T32 DK061918). PW acknowledges support from NIGMS (GM089564).

\section{References}

1. Nissen SE, Tsunoda T, Tuzcu EM, Schoenhagen P, Cooper CJ, Yasin M, Eaton GM, Lauer MA, Sheldon WS, Grines CL, Halpern S, Crowe T, Blankenship JC, Kerensky R (2003) Effect of recombinant ApoA-I Milano on coronary atherosclerosis in patients with acute coronary syndromes: a randomized controlled trial. JAMA 290, 2292-2300.

2. Gomaraschi M, Calabresi L, Franceschini G (2016) Protective Effects of HDL Against Ischemia/Reperfusion Injury. Front. Pharmacol. 7, 2. doi: 10.3389/fphar.2016.00002.

3. Schuler MA, Denisov IG, Sligar SG (2013) Nanodiscs as a new tool to examine lipidprotein interactions. Methods Mol. Biol. 974, 415-433.

4. Ryan RO (2008) Nanodisks: hydrophobic drug delivery vehicles. Expert Opin. Drug Deliv. 5, 343-351. 
5. Ghosh M, Ren G, Simonsen JB, Ryan RO (2014) Cationic lipid nanodisks as an siRNA delivery vehicle. Biochem. Cell Biol. 92, 200-205.

6. Cormode DP, Frias JC, Ma Y, Chen W, Skajaa T, Briley-Saebo K, Barazza A, Williams KJ, Mulder WJ, Fayad ZA, Fisher EA (2009) HDL as a contrast agent for medical imaging. Clin. Lipidol. 4, 493-500.

7. Panagotopulos SE, Witting SR, Horace EM, Maiorano JN, Davidson SW (2002) Bacterial expression and characterization of mature apolipoprotein A-I. Protein Expr. Purif. 25, 353-361.

8. Ryan RO, Forte TM, Oda MN (2003) Optimized bacterial expression of human apolipoprotein A-I. Protein Expr. Purif. 27, 98-103.

9. Tubb MR, Smith LE, Davidson WS (2009) Purification of recombinant apolipoproteins A-I and A-IV and efficient affinity tag cleavage by tobacco etch virus protease. J. Lipid Res. 50, 1497-1504.

10. Caparon MH, Rust KJ, Hunter AK, McLaughlin JK, Thomas KE, Herberg JT, Shell RE, Lanter PB, Bishop BF, Dufield RL, Wang X, Ho SV (2010) Integrated solution to purification challenges in the manufacture of a soluble recombinant protein in E. coli. Biotechnol. Bioeng. 105, 239-249.

11. Dagouassat N, Haeuw JF, Robillard V, Damien F, Libon C, Corvaïa N, Lawny F, Nguyen TN, Bonnefoy JY, Beck A (2001) Development of a quantitative assay for residual host cell proteins in a recombinant subunit vaccine against human respiratory syncytial virus. J. Immunol. Methods 251, 151-159.

12. Gursky O, Atkinson D (1996) Thermal unfolding of human high-density apolipoprotein A-1: implications for a lipid-free molten globular state. Proc Natl Acad Sci USA 93, 2991-2995.

13. Schulthess T, Schönfeld HJ, Seelig J (2015) Thermal unfolding of apolipoprotein A1. Evaluation of methods and models. Biochemistry 54, 3063-75.

14. Morrow JA, Segall ML, Lund-Katz S, Phillips MC, Knapp M, Rupp B, Weisgraber $\mathrm{KH}$ (2000) Differences in stability among the human apolipoprotein $\mathrm{E}$ isoforms determined by the amino-terminal domain. Biochemistry 39, 11657-11666.

15. Zhang L., Song J, Newhouse Y, Zhang S, Weisgrabe KH, Ren, G (2010)An Optimized Negative-staining Protocol of Electron Microscopy for apoE4.POPC Lipoprotein. J. Lipid Res, 51, 1228-1236. 
16. Zhang L., Song J, Cavigiolio G, Ishida BY, Zhang S., Kane JP, Weisgraber KH, Oda MN, Rye KA, Pownall HJ, Ren G (2011) The morphology and structure of lipoprotein revealed by optimized negative staining. J. Lipid Res. 52, 175-184.

17. Rames M, Yu Y, Ren G (2014) Optimized Negative Staining: a High-throughput Protocol for Examining Small and Asymmetric Protein Structure by Electron Microscopy. J. Vis. Exp. 90, e51087

18. Ludtke SJ, Baldwin PR, Chiu W (1999) EMAN: semiautomated software for highresolution single-particle reconstructions. J. Struct Biol. 128, 82-97.

19. Frank J, Radermacher M, Penczek P, Zhu J, Li Y, Ladjadj M, Leith A. (1996) SPIDER and WEB: processing and visualization of images in 3D electron microscopy and related fields. J. Struct Biol. 116, 190-199.

20. Grigorieff N (2007) FREALIGN: high-resolution refinement of single particle structures. J. Struct. Biol. 157, 117-125.

21. Ryan RO (2010) Nanobiotechnology applications of reconstituted high density lipoprotein. J. Nanobiotechnology. 8, 28. doi:10.1186/1477-3155-8-28.

22. Darabi M, Guillas-Baudouin I, Le Goff W, Chapman MJ, Kontush A (2016) Therapeutic applications of reconstituted HDL: When structure meets function. Pharmacol. Ther. 157, 28-42.

23. Brace RJ, Sorrenson B, Sviridov D, McCormick SPA (2010) A gel-based method for purification of apolipoprotein A-I from small volumes of plasma. J. Lipid Res. 51, 33703376.

24. Brewer HB, Ronan R, Meng M, Bishop C (1986) Isolation and characterisation of apolipoproteins A-I, A-II, and A-IV. Methods Enzymol. 128, 223-246.

35. Gröner A (2008) Pathogen safety of plasma-derived products - Haemate P/Humate-P. Haemophilia 14, Suppl 5, 54-71.

26. Feng MQ, Cai QS, Song DX, Dong JB, Zhou P (2006) High yield and secretion of recombinant human apolipoprotein Al in Pichia pastoris. Protein Expr. Purif. 46, 337342.

27. Sorci-Thomas MG, Parks JS, Kearns MW, Pate GN, Zhang C, Thomas MJ (1996) High level secretion of wild-type and mutant forms of human proapoA-I using baculovirus-mediated Sf-9 cell expression. J. Lipid Res. 37, 673-683. 
28. Pyle LE, Fidge NH, Barton PA, Luong A, Sviridov D (1997) Production of mature human apolipoprotein A-I in a baculovirus-insect cell system: propeptide is not essential for intracellular processing but may assist rapid secretion. Anal. Biochem. 253, 253-258.

29. Safi W, Maiorano JN, Davidson WS (2001) A proteolytic method for distinguishing between lipid-free and lipid-bound apolipoprotein A-I. J. Lipid Res. 42, 864-872.

30. Kanost MR, McDonald HL, Bradfield JY and Wyatt, GR (1987) Cloning and expression of the gene for apolipophorin III from Locusta migratoria. In: Molecular Entomology (JH Law, Ed) Alan R. Liss, Inc. 275-283.

31. Edelstein C, Scanu AM (1980) Effect of guanidine hydrochloride on the hydrodynamic and thermodynamic properties of human apolipoprotein Al in solution. $J$. Biol. Chem. 255, 5747-5754.

32. Serdakowski LA, Kerins B, Tschantz WR, Eisfeld J, Mackay K (2012) Endotoxin removal and prevention for pre-clinical biologics production. Biotechnol. J. 7, 1509-1516.

\section{Figure Legends}

Figure 1. Effect of culture temperature on apoA-I production. E. coli were transformed with a vector harboring the coding sequence of human apoA-I and cultured at $37^{\circ} \mathrm{C}, 30^{\circ} \mathrm{C}$ and $25^{\circ} \mathrm{C}$. Control bacteria were transformed with empty vector (EV) and cultured at $37^{\circ} \mathrm{C}$. Five $\mathrm{h}$ after induction with IPTG, bacteria were pelleted by centrifugation, re-suspended in PBS, lysed by probe sonication and centrifuged to separate insoluble material. The supernatant $(S)$ and pellet $(P)$ fractions from each culture condition were then subjected to SDS PAGE analysis. Far left lane: molecular weight standards; far right lane: apoA-I standard. A red box highlights the position of apoA-I in bacterial culture samples. The results shown are representative of 3 independent experiments.

Figure 2. Effect of heat treatment on bacterial lysate protein solubility. E. coli were transformed with a vector harboring the coding sequence of human apoA-I and cultured at $25^{\circ} \mathrm{C}$. The processed bacterial lysate supernatant was heated to the indicated 
temperatures for $10 \mathrm{~min}$ and cooled. Following this, samples were centrifuged to separate insoluble material and the resulting supernatant $(S)$ and pellet $(P)$ fractions analyzed by SDS PAGE. Far right lane: molecular weight standards; lane L: unprocessed bacterial lysate. The major band in each supernatant lane corresponds to apoA-I $(28 \mathrm{kDa})$. A red box highlights the migration position of apoA-I on the gel. The results shown are representative of 3 independent experiments.

Figure 3. Step-wise purification of recombinant untagged apoA-I. E. coli were transformed with an empty vector or a vector harboring the coding sequence of human apoA-I and cultured at $25^{\circ} \mathrm{C}$. The processed bacterial lysate supernatant was heated to $95^{\circ} \mathrm{C}$ for $10 \mathrm{~min}$. Following this, the sample was centrifuged to remove insoluble material and the supernatant applied to a reversed phase HPLC column. Aliquots were removed at various stages of the purification protocol for analysis. A) SDS PAGE; Lane 1: control E. coli lysate, Lane 2: apoA-I vector transformed $E$. coli lysate, Lane $3: 95^{\circ} \mathrm{C}$ exposure supernatant; Lane 4: apoA-I peak from HPLC; Lane 5: His-tag apoA-I standard. Molecular weight standards were applied to the far left lane. B) Anti human apoA-I Western blot; lane assignments same as panel A. The results shown are representative of 4 independent experiments.

Figure 4. Endotoxin levels. Endotoxin levels were measured by the Limulus Amebocyte Lysate assay at various stages of the purification scheme for His-tag apoA-I and untagged apoA-I. Values reported are the mean \pm S.D. $(n=3)$.

Figure 5. Secondary structure content and stability of apoA-I. A) Far UV CD spectra of His-tag apoA-I and untagged apoA-I. B) Both apoA-I proteins were incubated with increasing concentrations of guanidine $\mathrm{HCl}$ and the folded $\rightleftarrows$ unfolded transition midpoint determined from changes in molar ellipticity at $222 \mathrm{~nm}(\mathrm{~B})$. Results are presented as mean \pm S.D. $(n=3)$.

Figure 6. ApoA-I mediated cholesterol efflux. J774 macrophages were loaded with $\left[{ }^{3} \mathrm{H}\right]$ cholesterol followed by incubation with $10 \mu \mathrm{g}$ His-tag apoA-I or untagged apoA-I for 
specified times ranging from 1-24 h. Following incubation, the medium and cells were collected and radioactivity measured by liquid scintillation spectrometry. Results are reported as percent efflux: $\left[{ }^{3} \mathrm{H}\right]$ cholesterol in the media over total $\left[{ }^{3} \mathrm{H}\right]$ cholesterol (media + cell). Values are the mean \pm S.D. $(n=4)$.

Figure 7. Negative stain EM images and reference-free class averages of apoA-I rHDL. A) Survey view of rHDL prepared by optimized negative staining. B) Twenty-five representative rHDL particles. The first, second and third rows show the top, half-side and side views of the most abundant particle population ( 12 nm in diameter). The fourth and fifth rows show top and side views of second most abundant particle population ( $20 \mathrm{~nm}$ in diameter). C) Five selected reference-free class averages (from a total of $\sim 80$ classes) corresponding to representative particles from the left panel. 


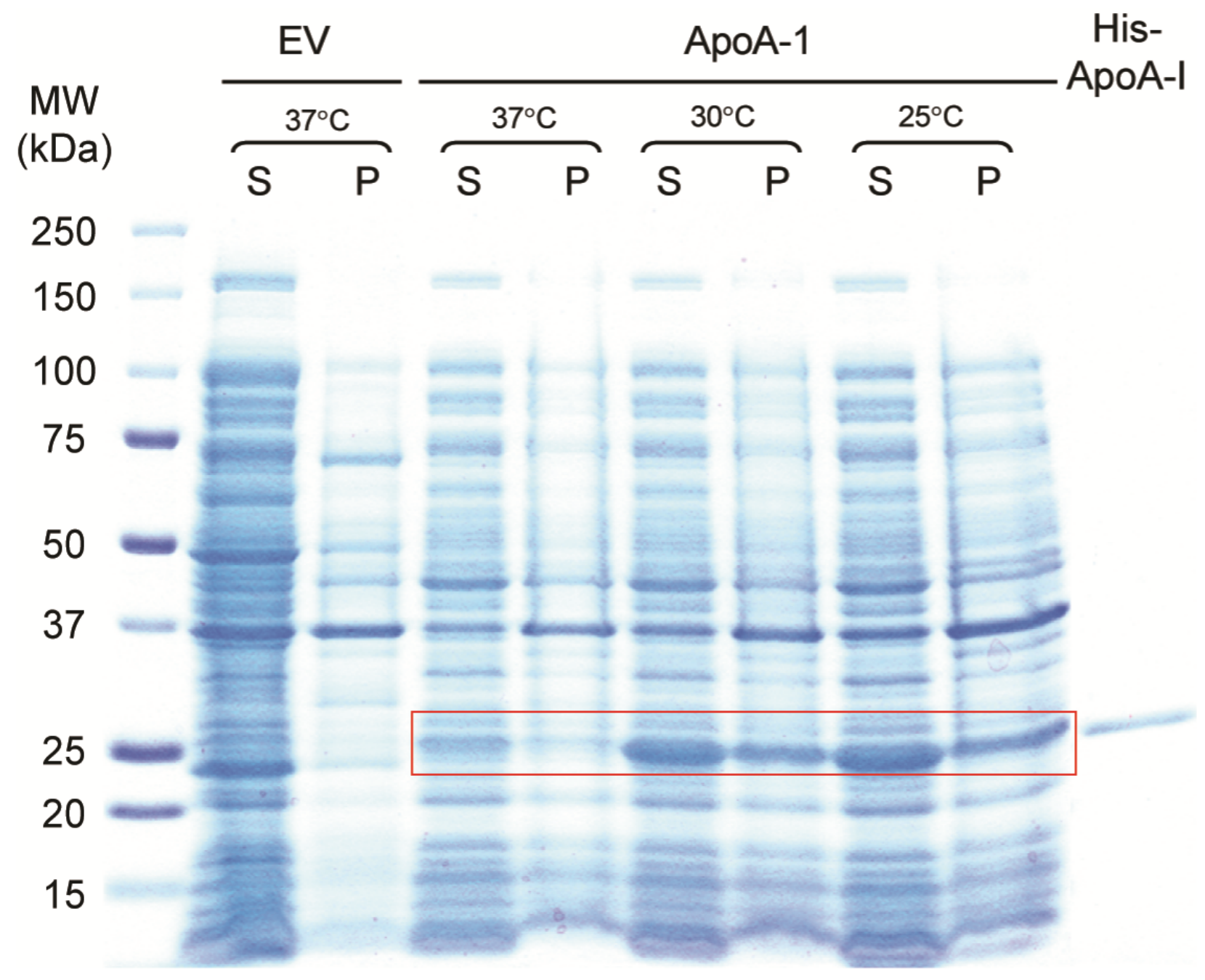




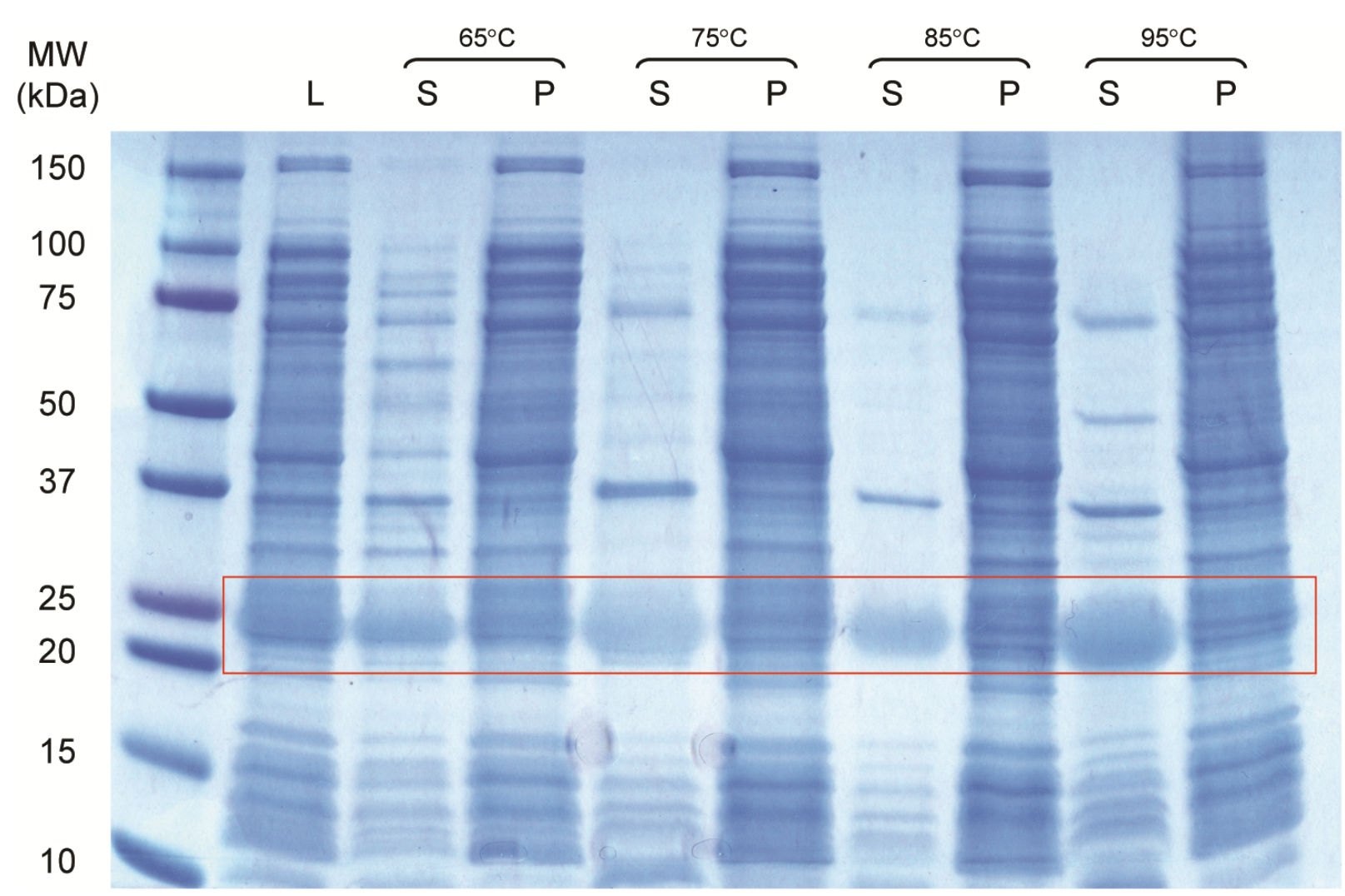


A

B
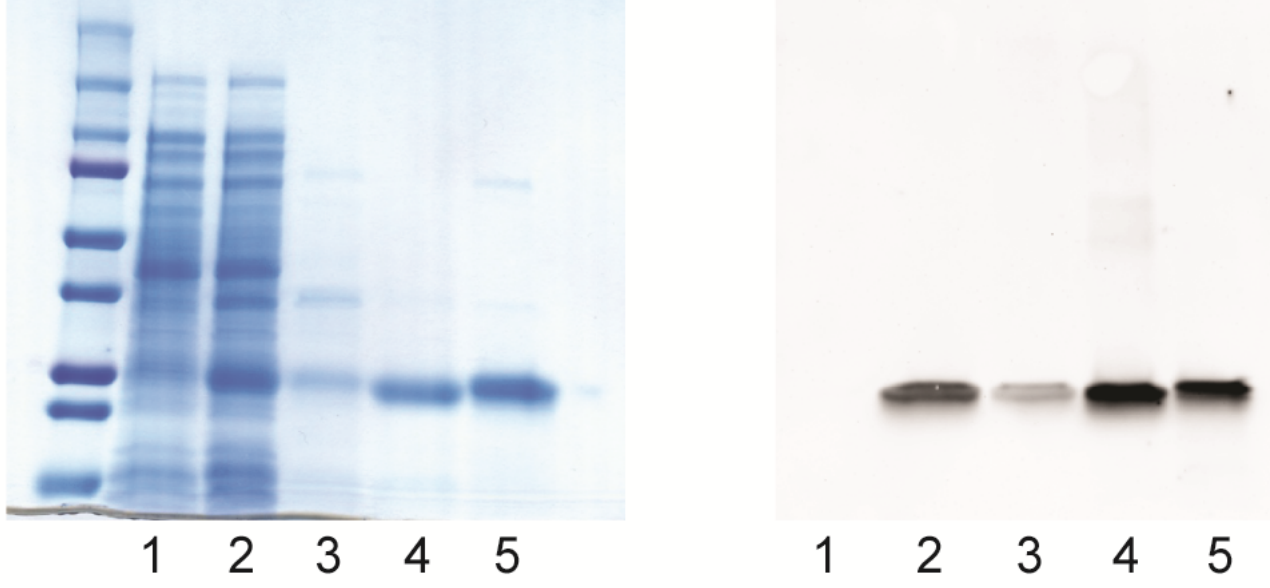


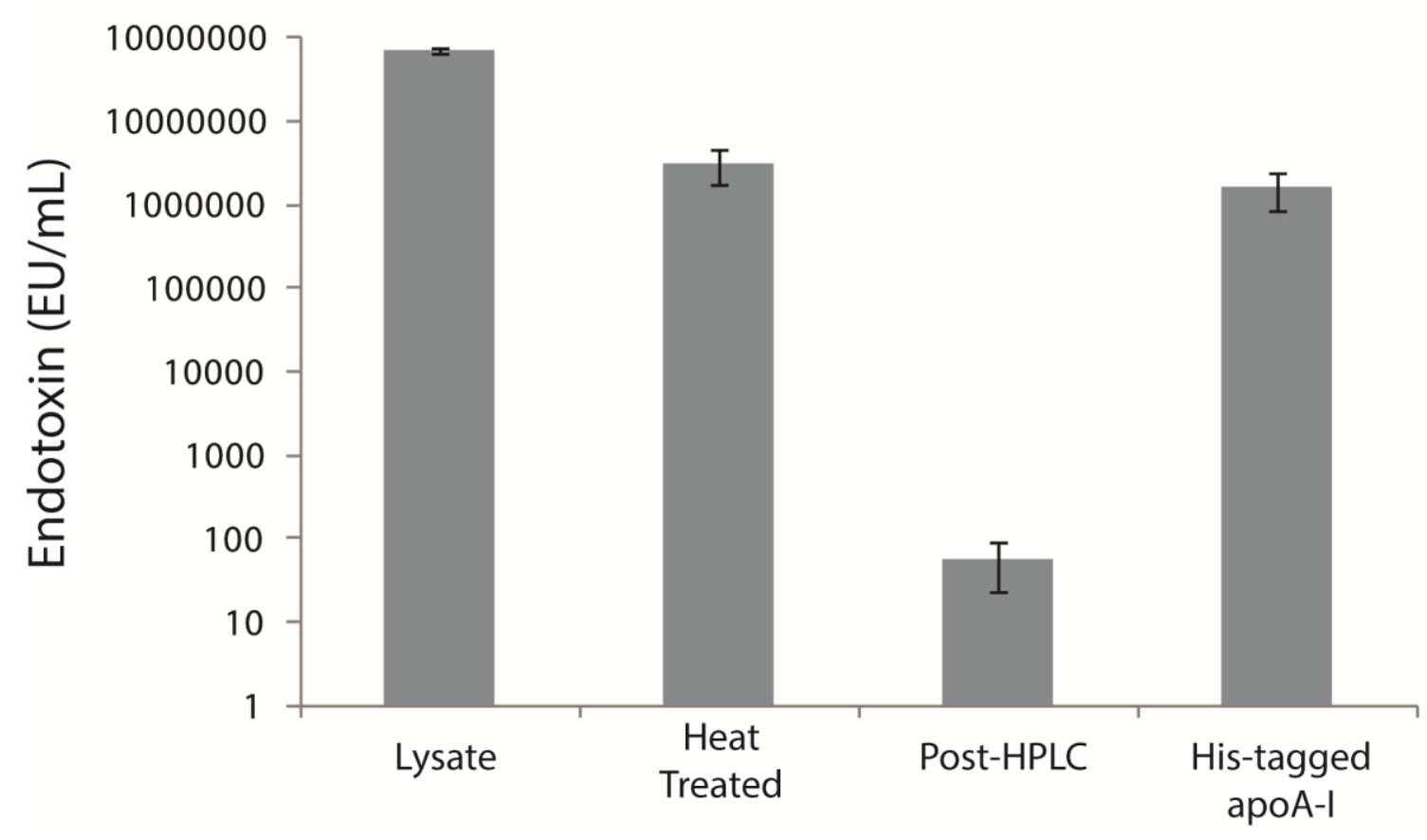


A

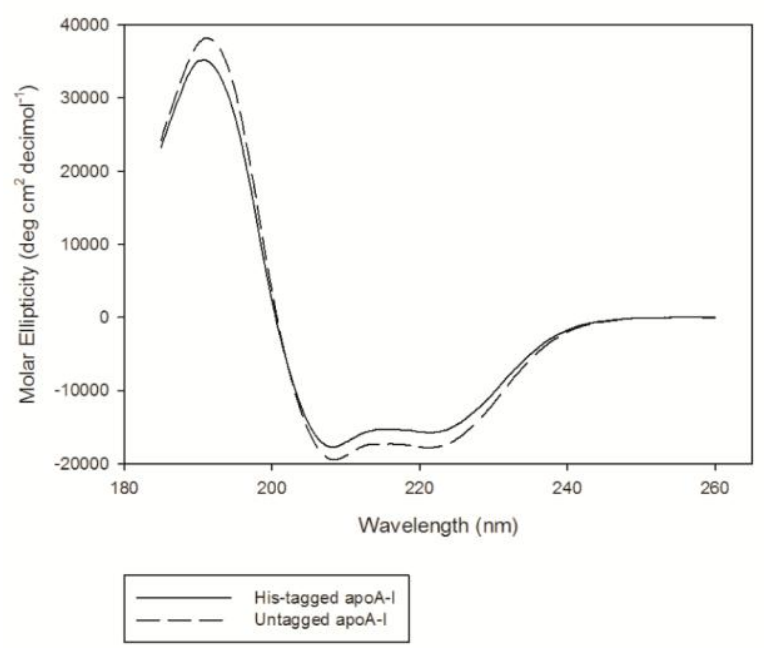

B

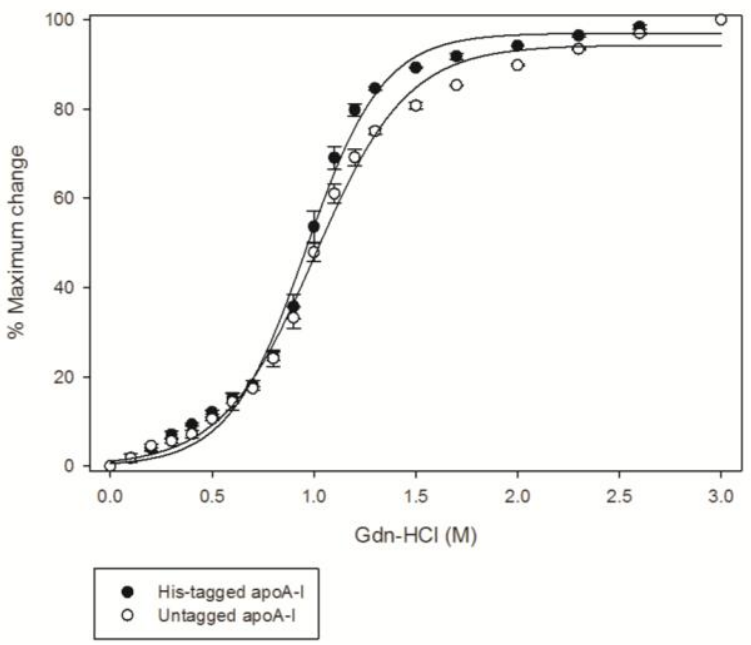




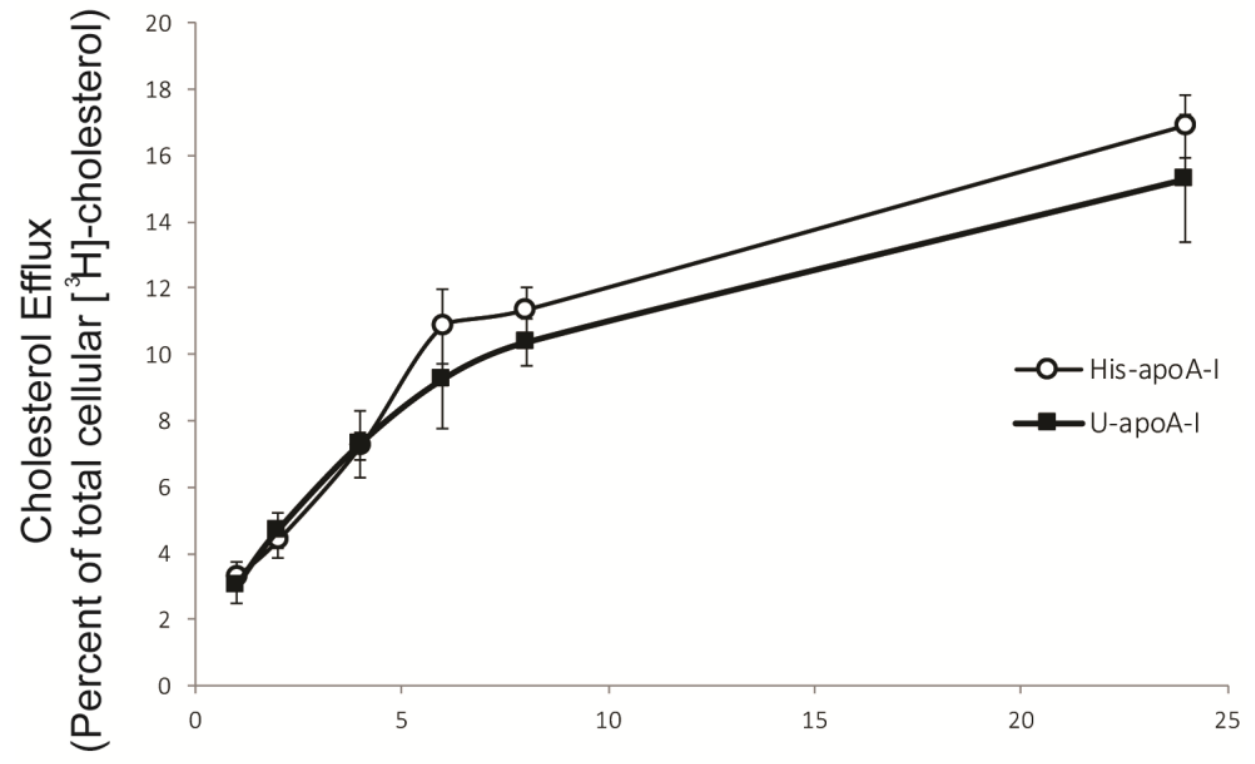

Time (h) 

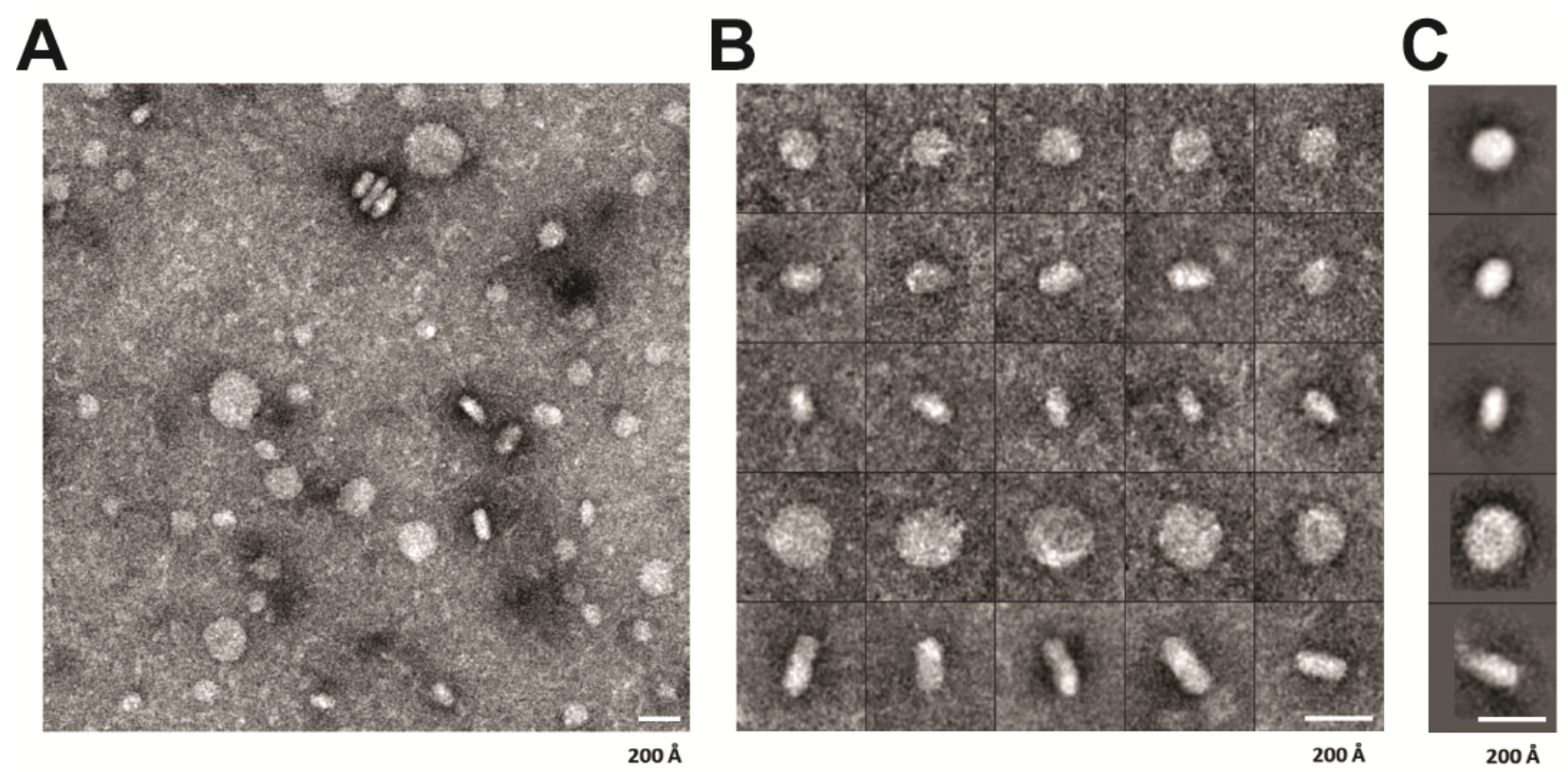